\title{
HIV-I Transgenic Rat Prefrontal Cortex Hyper-Excitability is Enhanced by Cocaine Self-Administration
}

\author{
Wesley N Wayman ${ }^{1,2}$, Lihua Chen ${ }^{1,2}$, Xiu-Ti Hu, ${ }^{*, 2}$ and T Celeste Napier ${ }^{1,2,3}$ \\ 'Department of Pharmacology, Rush University Medical Center, Chicago, IL, USA; ${ }^{2}$ Center for Compulsive Behavior and Addiction, Rush University \\ Medical Center, Chicago, IL, USA; ${ }^{3}$ Department of Psychiatry, Rush University Medical Center, Chicago, IL, USA
}

\begin{abstract}
The medial prefrontal cortex (mPFC) is dysregulated in HIV-I-infected humans and the dysregulation is enhanced by cocaine abuse. Understanding mPFC pathophysiology in this comorbid state has been hampered by the dearth of relevant animal models. To help fill this knowledge gap, electrophysiological assessments were made of mPFC pyramidal neurons (PN) from adult male HIV-I transgenic (Tg) F344 rats (which express seven of the nine HIV-I toxic proteins) and non-Tg F344 rats that self-administered cocaine for I4 days (COC-SA), as well as saline-yoked controls (SAL-Yoked) and experimentally naive Tg and non-Tg rats. Forebrain slices were harvested and prepared for whole-cell patch-clamp recording, and in treated rats, this occurred after |4- 8 days of forced abstinence. Aged-matched

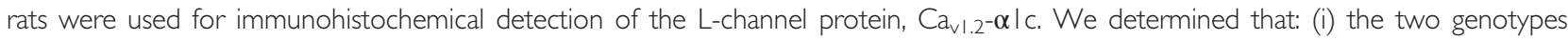
acquired the operant task and maintained similar levels of COC-SA, (ii) forced abstinence from COC-SA enhanced mPFC PN excitability in both genotypes, and neurons from Tg rats exhibited the greatest pathophysiology, (iii) neurons from SAL-Yoked Tg rats were more excitable than those from SAL-Yoked non-Tg rats, and in Tg rats (iv) blockade of L-type $\mathrm{Ca}^{2+}$ channels reduced the enhanced excitability,

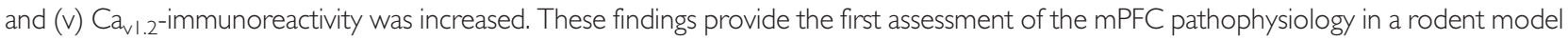
of HIV-I-mediated neuropathology with and without cocaine self-administration. Outcomes reveal an enhanced cortical excitability during chronic exposure to HIV-I proteins that is excessively exacerbated with cocaine abuse. Such neuropathophysiology may underlie the cognitive dysregulation reported for comorbid humans.

Neuropsychopharmacology (2016) 4I, 1965-1973; doi: I0.1038/npp.2015.366; published online I3 January 2016
\end{abstract}

\section{INTRODUCTION}

$\mathrm{HIV}^{+}$individuals remain at risk for developing HIVassociated neurological and psychological disorders despite suppressed viral replication provided by combination antiretroviral therapies (cART) (Tozzi et al, 2007). Symptoms include impaired concentration, memory deficits, and depression (Meyer et al, 2013; Gibbie et al, 2007). These may reflect a dysregulation of the prefrontal cortex (PFC) associated with HIV infection (Ferris et al, 2008; Thompson et al, 2005). Drug addiction is a frequent comorbidity with HIV infection (World Health Organization and Joint United Nations Programme on HIV/AIDS, 2009), and the functional pathology of the PFC seen in HIV-infected individuals is exacerbated by psychostimulant abuse (Meyer et al, 2013).

HIV is not thought to infect neurons, and HIV-associated neuropathology largely reflects the actions of neurotoxic viral proteins and proinflammatory molecules secreted by infected astrocytes and microglia (Gonzalez-Scarano and MartinGarcia, 2005; Hauser et al, 2007). These neuropathological

\footnotetext{
*Correspondence: Dr X-T Hu, Department of Pharmacology, Rush University Medical Center, 1735 W. Harrison Street, Cohn Research Building, Room 454, Chicago, IL 606/2, USA, Tel: + I 312563 4560, Fax: + I 312563 2403, E-mail: xiu-ti_hu@rush.edu

Received 7 June 2015; revised 14 November 2015; accepted 15 December 2015; accepted article preview online 18 December 2015
}

processes proceed even when viral replication is suppressed in the periphery by cART (Heaton et al, 2010, 2011). Several viral proteins are neurotoxic, including the envelope glycoprotein, gp120, an essential mediator of virus entry into host cells, and the transactivator of transcription (Tat), an activator of transcription of the viral genome (Jones et al, 1998; Haughey and Mattson, 2002). Tat excessively increases $\mathrm{Ca}^{2+}$ influx in neurons, including pyramidal neurons (PN) of the medial prefrontal (mPFC) (Brailoiu et al, 2008; Napier et al, 2014), by prolonging $\mathrm{Ca}^{2+}$ potentials mediated by L-type $\mathrm{Ca}^{2+}$ channels (Lchannels) that profoundly enhances firing (Napier et al, 2014). In vitro studies have demonstrated that neurotoxic effects of gp120 and Tat can be enhanced by other viral proteins (Nath et al, 2000) and cocaine (Aksenov et al, 2006). The consequences of chronic in vivo exposure to HIV-1 proteins on the MPFC, and whether COC-SA augments these effects, have not yet been determined.

The current study addressed this knowledge gap. We used non-infectious HIV-1 transgenic ( $\mathrm{Tg}$ ) rats, which express seven of the nine HIV-1 toxic proteins, including Tat and gp120 (Rao et al, 2011; Reid et al, 2001), to determine how chronic exposure to these proteins alters mPFC PN. To model human cocaine taking and addiction, we trained the rats to chronically self-administer the drug. To determine whether cocaine-mediated changes are detected even after 
drug-taking ceased, forebrain slices were obtained from these rats 14-18 days after the last operant session, and whole-cell patch-clamp electrophysiological recordings were conducted on mPFC PN. We determined that mPFC PN were dysregulated by the Tg state and by COC-SA and that pathophysiology was greater in the comorbid condition.

\section{MATERIALS AND METHODS}

\section{Animals}

Adult male non-transgenic Fischer F344 (non-Tg) and HIV-1 transgenic Fischer F344 (Tg) rats were purchased at 3-4 weeks of age (Harlan Labs, Indianapolis, IN) and housed two per cage under a 12-h light/dark cycle in an environmentally controlled facility at Rush University, with food and water provided ad libitum. For electrophysiological studies, one set of non- $\operatorname{Tg}(n=16)$ and $\operatorname{Tg}(n=11)$ was randomly assigned to COC-SA or SAL-Yoked treatment groups, and another set of non- $\mathrm{Tg}(n=2)$ and $\mathrm{Tg}(n=5)$ rats remained treatment naive. For immunohistochemistry, SAL-Yoked non- $\mathrm{Tg}(n=4)$ and $\mathrm{Tg}(n=6)$ rats taken from a parallel study were used. Rats were handled in accordance with the procedures established in the Guide for the Care and Use of Laboratory Animals (National Research Council, Washington DC) as approved by the Rush University IACUC.

\section{Procedures for Self-Administration Tasks}

Surgical instrumentation and self-administration tasks generally followed our published protocols (Wayman et al, 2015a). In brief, isoflurane-anesthetized rats were surgically fitted with an $11.5-\mathrm{cm}$ jugular vein catheter connected to a port externalized on the rats' back. Rats were allowed to recover for a minimum of 7 days and had to be at presurgical weight before behavioral training. Catheters were flushed daily with $0.1-0.2 \mathrm{ml}$ sterile saline. The operant chambers (Med-Associates, St Albans, VT) were fitted with two holes in which the rats could poke their nose. Upon a nose poke, a motor-driven syringe pump (Med-Associates) delivered infusions of saline or cocaine $(0.1 \mathrm{mg} / \mathrm{kg} / 0.1 \mathrm{ml})$ via tubing connected the rat's port. The concentration was based on our pilot studies wherein we determined that performance improved with concentrations below the $1.0 \mathrm{mg} / \mathrm{kg} /$ infusion we use for Sprague-Dawley rats (Wayman et al, 2015a) and that Fischer rats readily acquired and maintained COC-SA at $0.1 \mathrm{mg} / \mathrm{kg} / 0.1 \mathrm{ml}$ of cocaine. Test sessions were $2 \mathrm{~h}$ per day for 14 days. For COC-SA rats, the left hole was designated as 'active'. A cocaine infusion was delivered over $6 \mathrm{~s}$ each time the rat poked his nose in this hole (a fixed-ratio 1 schedule of reinforcement; FR1) and was accompanied by a tone and illumination of a cue light above the hole. During the infusion, additional nose pokes were recorded but had no programmed consequences. There was no post-infusion time-out period. Nose pokes in the inactive hole had no programmed consequences. For SAL-Yoked rats, both holes were inactive and neither the cue light nor the infusion pump was activated by nose pokes. After the last operant session, rats underwent 14-18 days of forced abstinence, during which they remained in their home cage. This allowed for
mPFC assessments from drug-free rats to better reflect the persistent maladaptations that occur in abstinent human cocaine abusers (Goldstein and Volkow, 2002).

\section{Ex vivo Electrophysiology}

Procedures followed our published protocols (Napier et al, 2014; Wayman et al, 2015a). To overview, after forced abstinence, rats (5.5-7 months of age) were anesthetized with chloral hydrate $(400 \mathrm{mg} / \mathrm{kg}$, i.p.), the brains were excised, and immersed in ice-cold, solution (in mM; 248 sucrose, $2.9 \mathrm{KCl}, 2 \mathrm{MgSO}_{4}, 1.25 \mathrm{NaH}_{2} \mathrm{PO}_{4}, 26 \mathrm{NaHCO}_{3}, 0.1 \mathrm{CaCl}_{2}$, 10 glucose, 2.5 kynurenic acid, and 1.0 ascorbic acid; $\mathrm{pH}$ 7.4-7.45, with $335-345 \mathrm{mOsm})$. Coronal sections $(300 \mu \mathrm{m})$ containing the $\mathrm{mPFC}$ were sliced and transferred to artificial cerebrospinal fluid (aCSF; in mM; $125 \mathrm{NaCl}, 2.5 \mathrm{KCl}, 25$ $\mathrm{NaHCO}_{3}, 1.25 \mathrm{NaH}_{2} \mathrm{PO}_{4}, 1 \mathrm{MgCl}_{2}, 2 \mathrm{CaCl}_{2}$, and 15 glucose; $\mathrm{pH}$ 7.4, with $305-315 \mathrm{mOsm})$. After $1 \mathrm{~h}$ incubation at room temperature, slices were transferred to a recording chamber perfused with oxygenated aCSF at $\sim 34^{\circ} \mathrm{C}$. Heatpulled glass electrodes were filled with a solution (in $\mathrm{mM}$; $120 \mathrm{~K}$-gluconate, $10 \mathrm{HEPES}, 20 \mathrm{KCl}, 2 \mathrm{MgCl}_{2}, 3 \mathrm{Na}_{2} \mathrm{ATP}, 0.3$ NaGTP, 0.1 EGTA, 0.1\% biocytin; pH, 7.3-7.35; mOsm, $280-285)$ to provide $4-6 \mathrm{M} \Omega$. PN were visually identified (Olympus BX-51,Luigs and Neumann, Ratingen, Germany) within the prelimbic (PrL) mPFC subregion in layers 5/6. The PN electrophysiological profile matched that previously determined for biocytin-labeled PrL-mPFC PNs (Wayman et al, 2015a). Recorded signals were amplified and filtered (MultiClamp 700A, Molecular Devices, Union City, CA) and digitized (Digidata 1322, Axon Instruments, Molecular Devices, Union City, CA). Activity was evoked with $500 \mathrm{~ms}$ current pulses incremented in $+25 \mathrm{pA}$ steps between -500 to $+400 \mathrm{pA}$. Neuronal recordings from SAL-Yoked non-Tg rats had to show a stable resting membrane potential (RMP) that was more negative than $-63 \mathrm{mV} ; 10 / 10$ neurons recorded met these criteria. The criteria were not applied to neurons from $\mathrm{Tg}$ rats or cocaine-exposed rats as these frequently exhibited a more depolarized RMP (5/14 neurons from COC-SA non-Tg rats, 9/15 from SAL-Yoked Tg, and 1/18 for COC-SA Tg met criteria; eg, Table 1). Action potentials evoked by the rheobase had to exhibit an amplitude from the threshold to peak of $>60 \mathrm{mV}$. L-channels' involvement was verified by response antagonism with bath-applied diltiazem ( $40 \mathrm{nM}$; Sigma/RBI, St Louis, MO), a blocker of open L-channels (Glossmann et al, 1983). We previously determined that $40 \mathrm{nM}$ was sufficient to antagonize the effects of bath-applied Tat on isolated $\mathrm{Ca}^{2+}$ potentials without altering mPFC PN electrophysiological profiles when given alone (Napier et al, 2014).

\section{Immunohistochemistry}

Procedures for immunohistochemistry followed our published protocol (Wayman et al, 2012). To overview, chloral hydrate-anesthetized rats were perfused transcardially with ice-cold saline followed by $4 \%$ paraformaldehyde ( $\mathrm{pH}$ 7.4). Removed brains were postfixed in $4 \%$ paraformaldehyde overnight, transferred to $30 \%$ sucrose until saturated, sliced into serial coronal sections $(40 \mu \mathrm{m})$, and stored in cryoprotectant at $-20^{\circ} \mathrm{C}$. Primary antibody incubation was conducted with anti-Ca $\mathrm{va}_{\mathrm{v} .2}-\alpha \mathrm{lc}$ (pore-forming L-channel 
Table I Active and Passive Membrane Properties of Deep Layer PrL mPFC PN

\begin{tabular}{|c|c|c|c|c|}
\hline & SAL-Yoked & COC-SA & SAL-Yoked & COC-SA \\
\hline $\mathrm{RMP}(\mathrm{mV})$ & $-70.0 \pm 0.7$ & $-61.8 \pm 0.6^{\# \# \#}$ & $-63.6 \pm 1.0$ ******* & $-57.2 \pm 0.9$ ****, \#\#\# \\
\hline $\mathrm{R}_{\text {in }}(M \Omega)$ & $159.4 \pm 8.0$ & $|65.1 \pm 1| .3$ & $185.6 \pm 13.5$ & $200.0 \pm 12.6 *$ \\
\hline Peak amplitude (mV) & $82.2 \pm 3.7$ & $80.6 \pm 3.1$ & $78.8 \pm 2.1$ & $80.4 \pm 3.4$ \\
\hline $1 / 2$ Peak duration (ms) & $1.9 \pm 0.1$ & $2.2 \pm 0.2$ & $1.9 \pm 0.1$ & $2.1 \pm 0.1$ \\
\hline $\mathrm{AHP}(\mathrm{mV})$ & $-11.2 \pm 0.8$ & $-9.7 \pm 0.9$ & $-13.7 \pm 1.0$ & $-9.2 \pm 0.8^{\# \# \#}$ \\
\hline
\end{tabular}

Two-way ANOVA with post-hoc Newman-Keuls comparison.

${ }^{*} p<0.05$, **** $p<0.00$ I between non-Tg-SAL and Tg-SAL, non-Tg-COC, and Tg-COC, respectively.

$\#$ \#\# $<0.0$ I, \#\#\# $p<0.00$ I between non-Tg-SAL and non-Tg-COC, Tg-SAL, and Tg-COC, respectively.

subunit) ( $1: 100$; Alomone Labs, Jerusalem, Israel) for $24 \mathrm{~h}$ at room temperature followed by the secondary antibody BA-1000 ( 1 : 200; Vector Laboratories Inc, Burlingame, CA). $\mathrm{Ca}_{\mathrm{v} 1.2}-\alpha 1 \mathrm{c}$ immunoreactivity $\left(\mathrm{Ca}_{\mathrm{v} 1.2}\right.$-ir) was visualized using a chromogen solution containing 3'3-diaminobenzidine and hydrogen peroxide enhanced with nickel. Absence of primary antibody served as negative controls. Stereological estimates of $\mathrm{Ca}_{\mathrm{v} 1.2}$-ir by a treatment-blind observer followed our published protocol (Wayman et al, 2012). In brief, the Stereo Investigator 2000 system (MBF Bioscience, Williston, VT) was used for unbiased stereological estimates per an optical fractionator procedure. Under low magnification $(\times 2)$, the regions of interest were outlined. Optical dissectors were defined using dissector height set at $25 \mu \mathrm{m}$ and guard zones of $2 \mu \mathrm{m}$. Cell counts were made in a counting frame of $75 \mu \mathrm{m}^{2}$ at regular intervals $(X=250 \mu \mathrm{m}, Y=250 \mu \mathrm{m})$ and analyzed using a $\times 20$ objective. The coefficient of error was calculated using the Gundersen method (McBride et al, 2003).

\section{Statistical Analysis}

Data were analyzed using Graphpad Prism v5 (GraphPad Software, La Jolla, CA) and SigmaPlot v12 (Systat Software, San Jose, CA). Stereological estimations were compared between the naive non-Tg and $\mathrm{Tg}$ groups using unpaired $t$-test. Chi-squared tests were used for non-parametric comparison of spiking inhibition, rebound spiking, and spontaneous firing. Other comparisons involved an analysis of variance (ANOVA). Current-spike responses were analyzed by mixed model ANOVA factoring genotype (non-Tg or $\mathrm{Tg}$ ), drug treatment (saline or cocaine), and current. Membrane properties were analyzed by two-way ANOVA factoring genotype (non-Tg or Tg) and chronic treatment (saline or cocaine). Current-spike responses were analyzed by mixed model ANOVA factoring genotype, in vitro treatment (with/without diltiazem), and current. Significant ANOVA main effects and interactions were further evaluated with Newman-Keuls. Differences were considered significant with $\alpha<0.05$. Data are presented as mean and SEM.

\section{RESULTS}

\section{Cocaine Self-Administration}

Fourteen rats (non-Tg, $n=8 ; \mathrm{Tg}, n=6$ ) acquired the COC-SA protocol, and the number of nose pokes in the active hole was significantly greater than inactive nose pokes for both genotypes $\left(\mathrm{F}_{(1,23)}=24.985, p<0.001\right.$; Figure 1a); therefore, the rats associated the active hole with cocaine. Acquisition of the COC-SA protocol improved over test days $\left(\mathrm{F}_{(13,156)}=4.21, \quad p<0.01\right)$, but there was no statistical difference between genotypes. The number of active nose pokes (Figure 1a) and infusions (Figure 1b) were not influenced by genotype and the rats self-titrated $\sim 1.0 \mathrm{mg} /$ $\mathrm{kg}$ cocaine per day. SAL-Yoked controls (non-Tg, $n=8$; Tg, $n=5$, one $\mathrm{Tg}$ rat died) received equal number of saline infusions as their COC-SA counterparts. In both genotypes, rats that received cocaine exhibited a shorter latency to engage the active hole and receive the first infusion, as compared with SAL-Yoked rats $\left(\mathrm{F}_{(1,23)}=39.596, p<0.001\right)$; however, there was no significant difference in latency to first response between genotypes within the COC-SA or SAL-Yoked groups (COC-SA non-Tg $90 \pm 221 \mathrm{~s}$; COC-SA Tg $54 \pm 259$ s; SAL-Yoked non-Tg $1593 \pm 221$ s; SAL-Yoked Tg $1647 \pm 280 \mathrm{~s})$.

\section{COC-SA and the Tg State Enhanced Excitability of mPFC PNs}

The electrophysiological profile of deep layer PrL-mPFC PN is overviewed in Table 1. Regarding passive membrane properties, the RMP differed between genotype $\left(\mathrm{F}_{(1,53)}=\right.$ 42.447, $p<0.001)$ and treatment $\left(\mathrm{F}_{(1,53)}=74.784, p<0.001\right)$, but there was no interaction. Post-hoc evaluations revealed that neurons from COC-SA non-Tg rats were more depolarized compared with the RMP of neurons from SAL-Yoked non-Tg rats $(p<0.001)$, as were neurons from COC-SA Tg $v$ COC-SA non-Tg $(p<0.001)$, and SAL-Yoked Tg vs SAL-Yoked non-Tg $(p<0.001)$. $R_{\text {in }}$ was altered by genotype $\left(F_{(1,53)}=5.793, p=0.02\right)$ but not by treatment. Post-hoc analysis indicated that the genotype effect on the $R_{\text {in }}$ reflected neurons from COC-SA Tg rats ( vs COC-SA non-Tg 

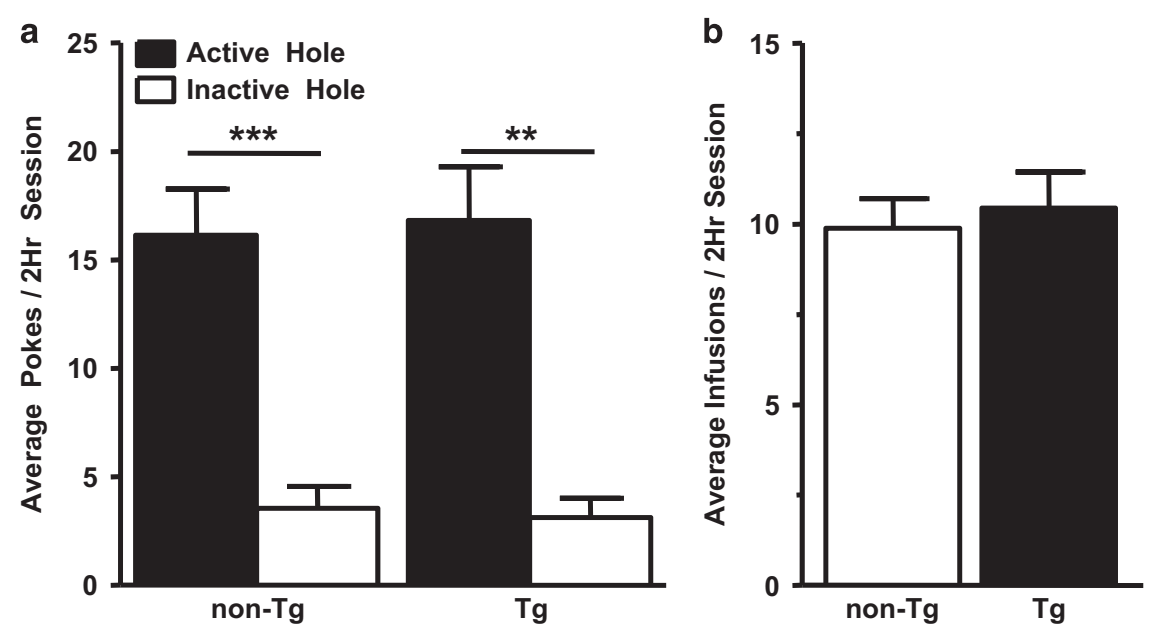

Figure I Both non- $\mathrm{Tg}$ and $\mathrm{Tg}$ rats performed the cocaine-self administration task. (a) Shown are the average number of daily nose pokes across the I4 once-daily sessions by non-Tg and Tg rats for cocaine self-administration. Two-way ANOVA with post-hoc Newman-Keuls revealed that significantly more nose pokes occurred in the active hole than in the inactive hole for both genotypes (*** $<0.01$; **** $p<0.00 \mathrm{I}$ ), illustrating that both genotypes learned to associate the active hole nose poking with cocaine reinforcement. Not illustrated are nose pokes by SAL-Yoked non-Tg and SAL-Yoked Tg in the active holes, which were $5.0 \pm 0.6$ and $6.8 \pm 1.6$, respectively $(p>0.05)$, or in the inactive holes, which were $3.5 \pm 1.0$ and $7.1 \pm 2.2$, respectively. (b) There was no significant difference in number of daily cocaine infusions averaged across 14 days between non- $\operatorname{Tg}$ and $\mathrm{Tg}$ rats.

rats; $p<0.05)$. Regarding active membrane properties, the rheobase was altered by genotype $\left(\mathrm{F}_{(1,53)}=13.838, p<0.001\right)$ and treatment $\left(\mathrm{F}_{(1,53)}=18.830, p<0.001\right)$, but there was no interaction. Post-hoc analysis revealed a lower rheobase in COC-SA non-Tg rats relative to SAL-Yoked non-Tg rats $(p<0.01)$, as well as for COC-SA Tg vs COC-SA non-Tg $(p<0.05)$ and SAL-Yoked Tg rats $v s$ SAL-Yoked non-Tg rats $(p<0.001)$. The AHP was not affected by genotype but was changed by treatment $\left(\mathrm{F}_{(1,53)}=10.708, p=0.002\right)$, with neurons from COC-SA Tg rats exhibiting reduced AHPs relative to SAL-Yoked $\mathrm{Tg}$ rats $(p<0.001)$. In summary, significant main effects were obtained for RMP, $R_{\text {in }}$, rheobase, and AHP, but no interactions occurred indicating that consequences of genotype and COC-SA were not synergistic. Significant post-hoc analyses within significant main effects suggest that effects could be additive. Correlation statistics did not indicate an association between amount of cocaine intake and neuronal membrane properties of either genotype (data not shown).

The increase in RMP and reduction in rheobase suggested that the basal state of PN differed between the genotypes and with COC-SA. Accordingly, we evaluated basal state spiking using the $500-\mathrm{ms} 0-\mathrm{pA}$ trial of the stimulation currentevoked response protocol. Some neurons from COC-SA non-Tg and COC-SA Tg rats exhibited spontaneous action potentials during this brief sample time; however, spontaneous firing did not occur in any neurons from SAL-Yoked rats (non-Tg or $\mathrm{Tg}$ ) (Figure 2a and b). Thus basal excitability was enhanced by a cocaine history in both genotypes.

Overexcitation by large depolarizing currents can reduce spike amplitude, and if sufficiently strong, spiking decreases, a phenomenon often referred to as 'depolarization-induced inactivation' (Grace and Bunney, 1986). Using this physiological readout as an index of spiking capacity, we evaluated depolarizing current pulses up to $+400 \mathrm{pA}$. A robust reduction in spike amplitude and number was often obtained with currents $>+200 \mathrm{pA}$ following COC-SA in both genotypes and also in $\mathrm{Tg}$ rats that were SAL-Yoked (Figure 2c). This phenomenon did not occur in any neurons from SAL-Yoked non-Tg rats (Figure 2c). But, as the COC-SA Tg group did not differ from any other group (Figure 2d), it appears that genotype or cocaine treatment was sufficient to promote this dysfunctional state.

At currents from $-25 \mathrm{pA}$ to $200 \mathrm{pA}$, the current-voltage relationship was linear for all treatment groups, and the curves were parallel with shifts that simply mirrored the group differences in RMP. Large hyperpolarizing currents (from $-500 \mathrm{pA}$ to $-300 \mathrm{pA}$ ) produced a slight inward rectification, but as these levels are not normally encountered by neurons, the data were not analyzed. At currents from -500 to $-25 \mathrm{pA}$, a significant proportion of neurons from $\mathrm{Tg}$ rats and COC-SA rats of both genotypes presented a post-hyperpolarization membrane rebound that resulted in spiking (Figure $2 \mathrm{e}$ and $\mathrm{f}$ ). Rebound spiking did not occur in any neurons from SAL-Yoked non-Tg rats. These findings suggest that subthreshold excitability is increased in neurons of Tg rats, as well as in both genotypes after chronic COC exposure.

Modest depolarizing currents (from $+25 \mathrm{pA}$ to $+100 \mathrm{pA}$ ) evoked firing that differed for genotype $\left(\mathrm{F}_{(1,212)}=40.281\right.$, $p<0.001)$, treatment $\left(\mathrm{F}_{(1,212)}=13.364, p<0.001\right)$, and current $\left(\mathrm{F}_{(3,212)}=62.349, p<0.001\right)$ (Figure 3 ), but there were no interactions. When comparing genotype effects within SAL-Yoked groups, these depolarizing currents evoked significantly more spikes in neurons from $\mathrm{Tg}$ rats relative to those from non- $\mathrm{Tg}$ rats $\left(\mathrm{F}_{(1,23)}=4.755, \quad p<0.05\right.$; Figure $3 \mathrm{~b}$ ). This genotype difference was not observed with higher currents (ie, from $+125 \mathrm{pA}$ to $+200 \mathrm{pA}$; see Figure $3 \mathrm{~b}$, insert), and many neurons exhibited features of overexcitation (eg, depolarization inactivation); therefore, these data were analyzed separately and were not included in evaluations of current-spike relationships (see below). There was a treatment effect within the $\mathrm{Tg}$ rats $\left(\mathrm{F}_{(1,93)}=9.871, p<0.01\right)$ and within non-Tg rats $\left(\mathrm{F}_{(1,66)}=4.696, p<0.05\right)$; spiking of neurons from COC-SA rats was significantly greater than those from SAL-Yoked rats in both genotypes. There was 


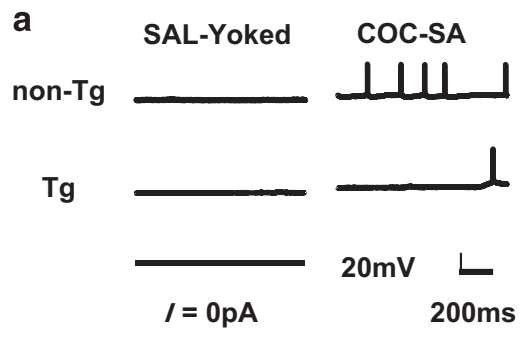

b

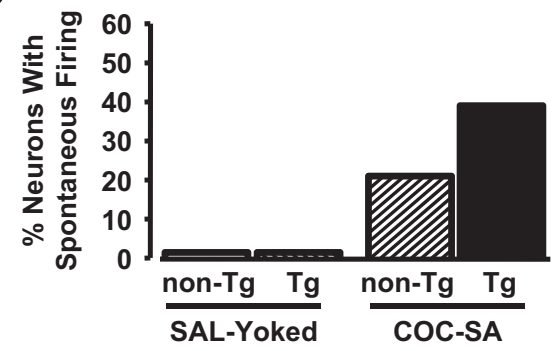

C

d
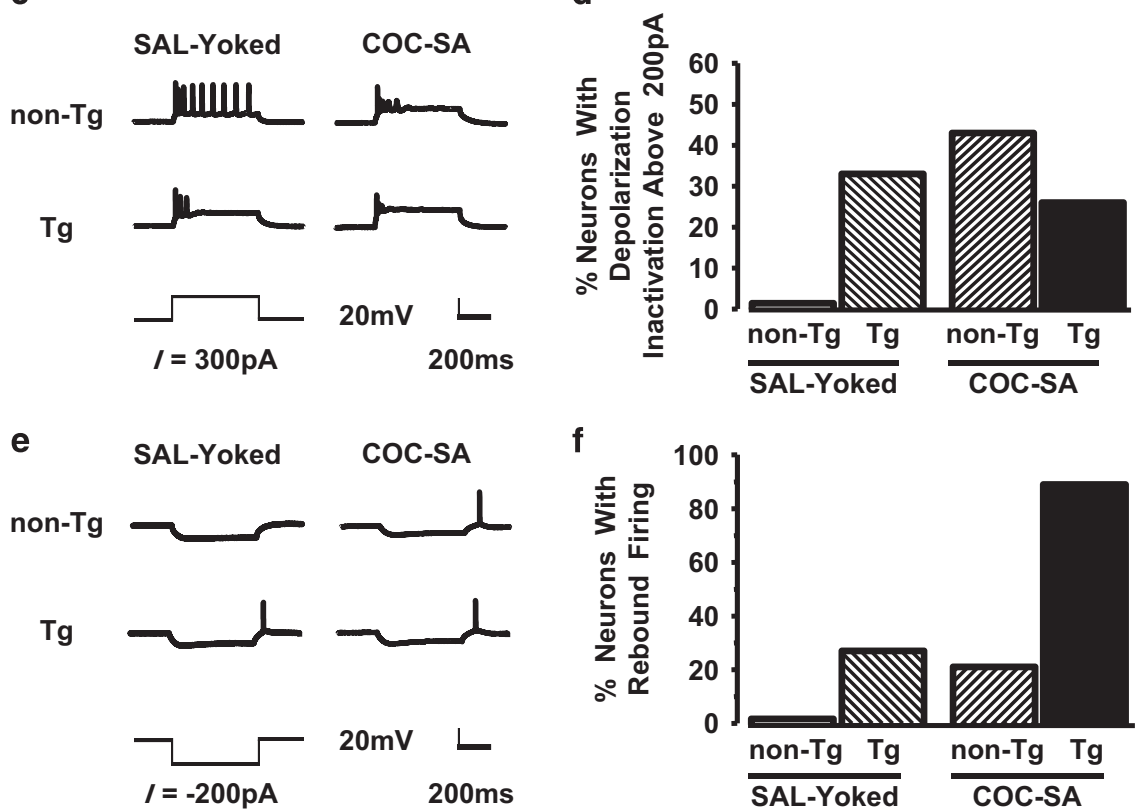

Figure 2 Spontaneous firing, over-excitation, and rebound firing were associated with cocaine self-administration. (a and b) Spontaneous firing. (a) Basal activity was not observed in neurons from SAL-Yoked non-Tg rats (top left) or SAL-Yoked Tg rats (bottom left), but spontaneous (non-evoked) spiking did occur in neurons from COC-SA non-Tg (top right) and COC-SA Tg (bottom right) rats. (b) Chi-square analysis revealed distribution differences for spiking number $\left(\chi_{(3)}^{2}=11.13 ; p<0.05\right)$ consistent with an increase in the propensity of neurons to exhibit spontaneous firing in rats that self-administered cocaine. (c and d) Overexcitation. (c) Traces of normal action potentials evoked in a neuron from a SAL-Yoked non-Tg rat (top left). The same current level evoked spiking with reduced action potential amplitudes and firing number in a neuron from a SAL-Yoked Tg rat (bottom left), as well as a neuron from a COC-SA non-Tg rat (top right) and COC-SA Tg rat (bottom right). This profile is consistent with over-excitation leading to depolarization inactivation. (d) The inactivation profile did not occur in any of the neurons from SAL-Yoked non-Tg rats. Nevertheless, because of a relatively balanced distribution among the other treatment groups, Chi-square analysis was not significant $\left(\chi_{(3)}^{2}=5.63, p=0.1313\right.$ ). (e and $\left.f\right)$ Rebound firing. Representative traces showing that a modest current $(-200 \mathrm{pA})$ that induced membrane hyperpolarization did not result in rebound firing in a neuron from a SAL-Yoked non-Tg rat (top left), but was sufficient to elicit spiking in neurons from a SAL-Yoked Tg rat (bottom left), COC-SA non-Tg rat (top right), and COC-SA Tg rat (bottom right). (f). Chi-square analysis revealed significant differences in the distribution of rebound spiking $(\chi(3)=27.63, p<0.00 \mathrm{I})$ consistent with an increase in the propensity of neurons in COC-SA Tg rats to exhibit $V_{m}$ rebound-elicited spiking.

a current effect for neurons from $\mathrm{Tg}$ rats $\left(\mathrm{F}_{(3,93)}=255.600\right.$, $p<0.001)$. Post-hoc analysis revealed significant differences at currents from +50 to $100 \mathrm{pA}$ between neurons from COC-SA Tg and SAL-Yoked Tg rats. There was also a current effect for neurons from non- $\mathrm{Tg}$ rats $\left(\mathrm{F}_{(1,66)}=38.182\right.$, $p<0.001)$ with significant differences between neurons from COC-SA non-Tg and SAL-Yoked non-Tg rats at +75 to $100 \mathrm{pA}$.

\section{The Status of L-type $\mathrm{Ca}^{2+}$ Channels}

The electrophysiological profile of mPFC $\mathrm{PN}$ from $\mathrm{Tg}$ rats paralleled that which we previously observed with the HIV-1 Tat-exposed mPFC PN in Sprague-Dawley rats wherein we also revealed that isolated L-potentials were enhanced
(Napier et al, 2014). Accordingly, additional experiments were conducted here to determine whether (i) the profile indeed involved L-channels by testing ability of a selective L-channel antagonist, diltiazem, to mitigate electrophysiological readouts of PN function, and (ii) L-channel protein levels were increased in $\mathrm{Tg}$ rats $v s$ non-Tg rats by immunohistochemically staining for the L-channel $\mathrm{Ca}_{\mathrm{v} 1.2}-\alpha 1 \mathrm{c}$ protein (termed $\mathrm{Ca}_{\mathrm{v} 1.2}$-ir). Firing of $\mathrm{mPFC} \mathrm{PN}$ from Tg rats $(n=7$ neurons from 5 rats) was reduced with bath-perfused diltiazem (Figure $4 \mathrm{a}$ and b). Diltiazem $\left(\mathrm{F}_{(1,42)}=10.067\right.$, $p<0.05)$ and depolarizing currents $\left(\mathrm{F}_{(7,42)}=29.117\right.$, $p<0.001)$ both altered spiking, but there was no interaction of these effects. Post-hoc analysis revealed a significant effect of diltiazem on evoked spiking for currents from +150 to $+200 \mathrm{pA}$ in Tg rats. In contrast, firing of mPFC PN from 


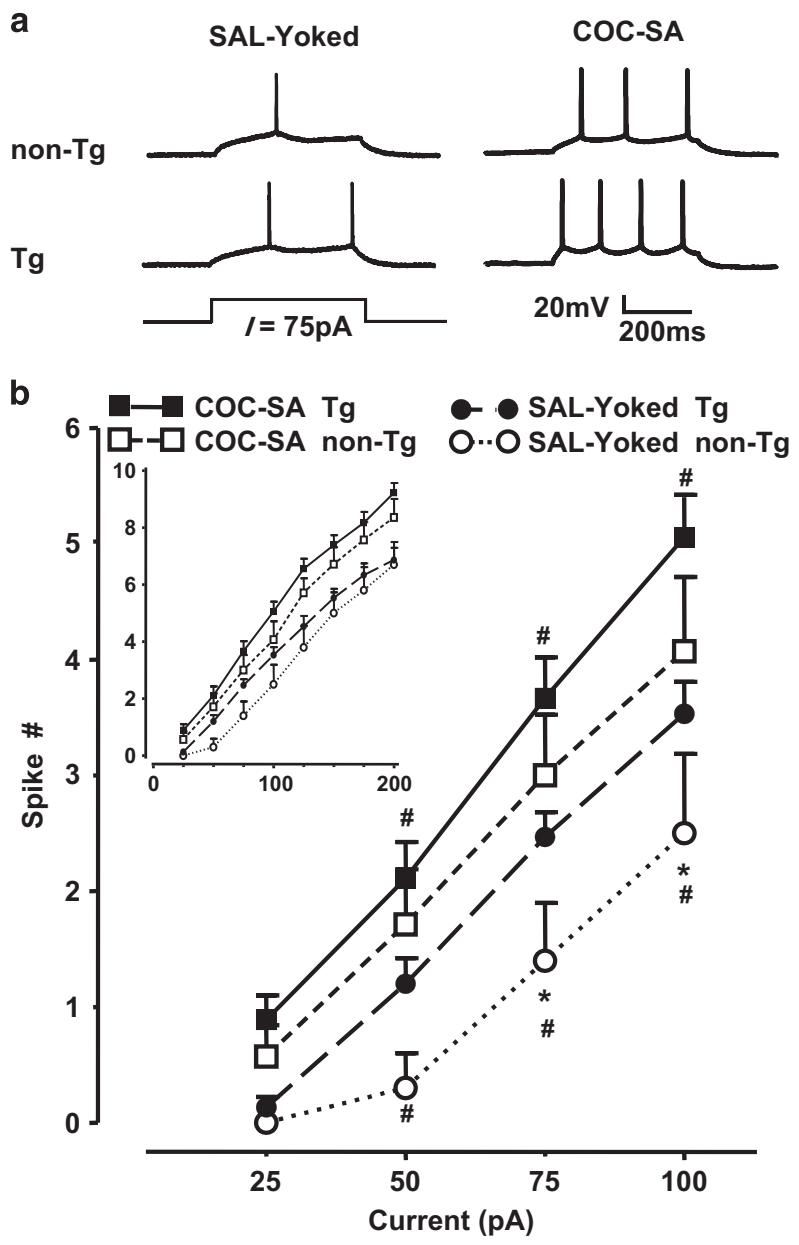

Figure 3 COC-SA enhanced firing of mPFC pyramidal neurons from non-Tg and $\mathrm{Tg}$ rats. (a) Individual traces of responses to a small (75 pA) depolarizing current pulse in mPFC pyramidal neurons from non-Tg (top row) and Tg (bottom row) rats in the SAL-Yoked (left) or COC-SA (right) treatment groups. (b) Illustrated is the relationship between depolarizing current magnitude $(25-100 \mathrm{pA})$ and the number of evoked spikes. Mixed model repeated-measures ANOVA with post-hoc Newman-Keuls $(p<0.05)$ revealed significant differences between COC-SA non-Tg rats and SAL-Yoked non-Tg rats at +50 to 100 pA (\#), COC-SA Tg rats and SAL-Yoked Tg rats at +50 to 100 pA (\#), and SAL-Yoked Tg and non-Tg rats at +75 to $100 \mathrm{pA}(*)$. There was no significant difference in the number of evoked spikes between neurons from Tg and non-Tg rats after COC-SA. Insert: Current-evoked spike relationships for depolarizing current pulses up to $+200 \mathrm{pA}$

non-Tg rats ( $n=5$ neurons from 2 rats) was not affected by diltiazem (Figure $4 \mathrm{a}) . \mathrm{Ca}_{\mathrm{v} 1.2}$-ir was readily detected in cells in layer 5/6 of the PrL mPFC in all rats. The cells were approximately $35 \mu \mathrm{m}$ in diameter with a distinct nucleus, a profile that is consistent for PN. Staining pattern was genotype specific. First, $\mathrm{Ca}_{\mathrm{v} 1.2^{-i r}}$ in non-Tg neurons was largely confined to cytoplasmic membranes. The cytoplasmic membranes from $\mathrm{Tg}$ neurons typically showed more intense staining, which was also seen in the cytoplasm (Figure $4 \mathrm{~b}$ ). Second, stereological assessments of layer 5/6 revealed the PrL mPFC of Tg rats displayed a significantly higher number of $\mathrm{Ca}_{\mathrm{v}} 1.2$-ir cells compared with non-Tg rats (Figures $4 \mathrm{c}$; $\left.t_{(8)}=5.683, p=0.001\right)$. These findings indicate that $\mathrm{Tg}$ rats overexpress L-channels in PN.
Chronic stress alters $\mathrm{Ca}^{2+}$ regulation in the mPFC (Wen et al, 2012) and the morphology of mPFC PN (for a review, see Kolb and Gibb, 2015). To ascertain whether surgical instrumentation and the 14 days of operant testing altered electrophysiological assessments of PN neurons of transgenic animals, we compared recordings from SAL-Yoked Tg rats to age-matched naive Tg rats. As shown in Figure 4a (insert), moderate depolarizing currents (from +25 to $+200 \mathrm{pA}$ ) evoked firing in MPFC PN of SAL-Yoked Tg rats with a magnitude that overlapped with that obtained from naive $\mathrm{Tg}$ rats. Likewise, the general electrophysiological profile of mPFC PN was similar between naive Tg and SAL-Yoked Tg rats (RMP: $-62.9 \pm 1.4$ vs $-63.6 \pm 1.0, R_{\text {in }}: 203.4 \pm 29.4 v s$ $185.6 \pm 13.5$, Rheobase: $71.4 \pm 19.2$ vs $55.0 \pm 3.6$, Threshold: $-42.9 \pm 2.1$ vs $-43.6 \pm 1.5$, Peak: $76.1 \pm 5.3$ vs $78.8 \pm 2.1,1 / 2$ Peak: $2.0 \pm 0.1$ vs $1.9 \pm 0.1$, AHP: $-13.1 \pm 0.9$ vs $-13.7 \pm 1.0$; respectively). These results suggest that the surgical instrumentation, repeated SAL infusions, or exposure to the self-administration operant task was not sufficient to alter the electrophysiological profile of the $\mathrm{PN}$ in $\mathrm{Tg}$ rats.

\section{DISCUSSION}

The current study describes mPFC pathophysiology associated with chronic exposure to $\mathrm{HIV}-1$ proteins and indicates an upregulation of L-channels as being involved in this endophenotype. This study also describes COC-taking behavior in $\mathrm{Tg}$ rats and the subsequent consequences on mPFC neuronal function. With the FR1 task, Tg rats acquired self-administration and maintained similar levels of cocaine intake, as did non-Tg rats. Following forced abstinence, PN excitability was enhanced in both genotypes, but SAL-Yoked $\mathrm{Tg}$ rats exhibited greater firing than SAL-Yoked non-Tg rats, and COC-SA Tg rats demonstrated the greatest excitability. These findings reveal pathophysiological interactions for cocaine abuse and chronic exposure to $\mathrm{HIV}-1$ proteins.

Fisher 344 non-Tg rats do not exhibit the robust drugtaking seen in other strains of rats, such as Lewis (Picetti et al, 2012; Meyer and Bardo, 2015) or Sprague-Dawley (Wayman et al, 2015a). Nonetheless, $0.1 \mathrm{mg} / \mathrm{kg}$ of cocaine/ infusion (ie, $\sim 33.3 \mu \mathrm{g} /$ infusion for $300 \mathrm{~g}$ rats) used in the current study was sufficiently motivating for both non-Tg and $\mathrm{Tg}$ rats to rapidly learn the operant task and to demonstrate a clear preference for the drug-paired hole relative to the inactive hole. Recently, McIntosh et al (2015) used three, 1-h components/operant session to study cocaine doses ranging from 5.2 to $333 \mu \mathrm{g} /$ infusion for $\mathrm{Tg}$ rats that weighed $260-285 \mathrm{~g}$. With this paradigm, cocaine preference was higher in $\mathrm{Tg}$ rats compared with non-Tg rats with 10.4 and $20.8 \mu \mathrm{g} /$ infusion, lower at $41.6 \mu \mathrm{g}$, and not different for 83.2-333.3 $\mu \mathrm{g} /$ infusion, so that there were no significant differences in the total lifetime intake between genotypes (McIntosh et al, 2015). Thus, as we report here, cocaine intake by $\mathrm{Tg}$ rats can be similar to that by non-Tg rats. Cocaine intake did not correlate with measured membrane properties or spiking profiles in either genotype. Moreover, as the functional deficits in mPFC PN were observed 14-18 days after the last operant session, the cocaine dose and self-administration protocol appear to be sufficient to underpin a persistent or delayed neuropathophysiology. 


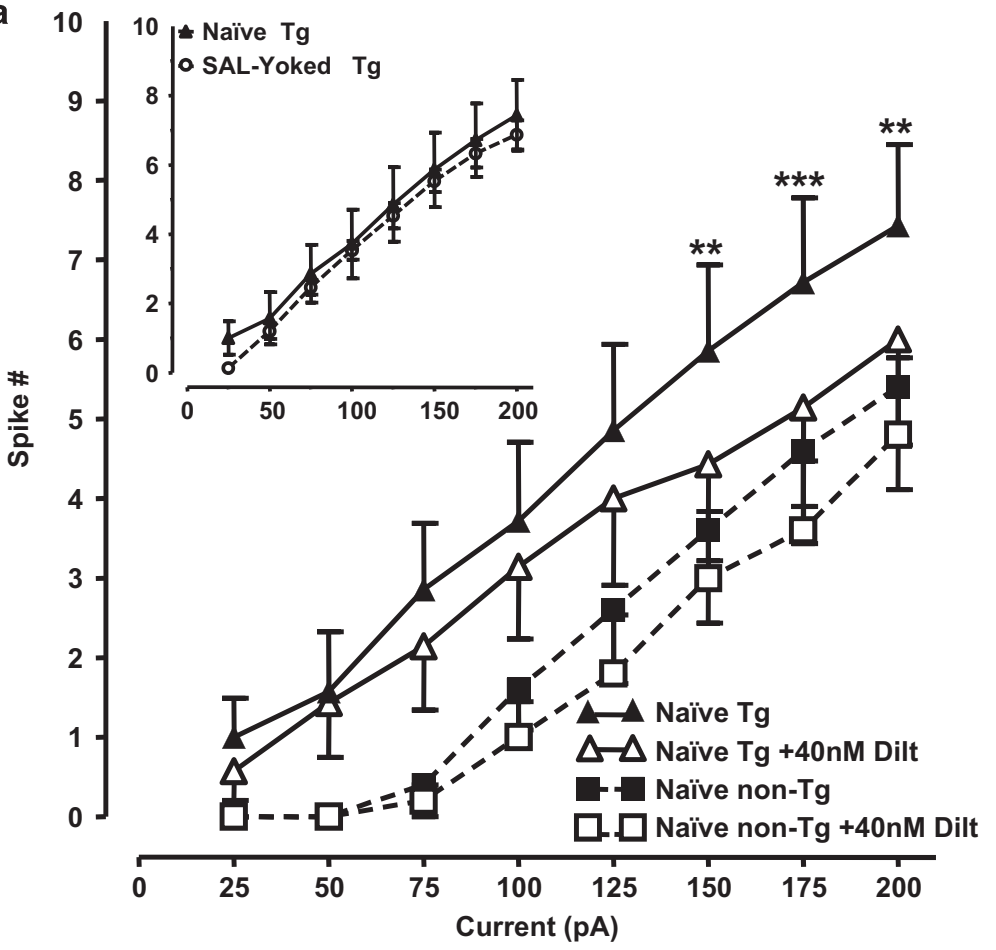

b

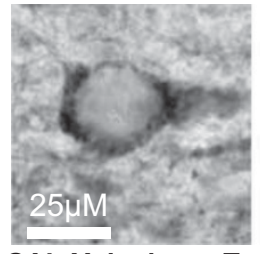

SAL-Yoked non-Tg

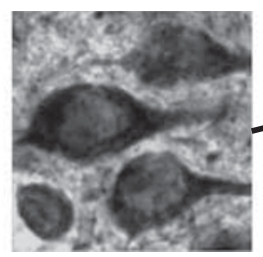

SAL-Yoked Tg
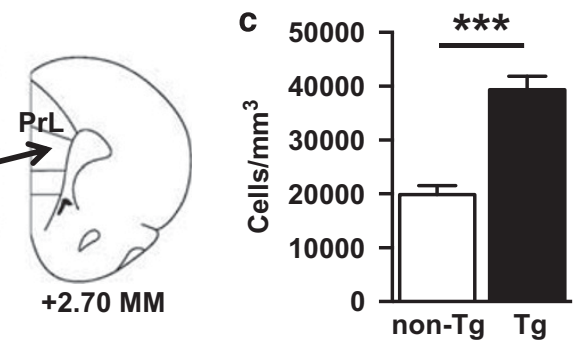

Figure 4 L-channel blockade reduced evoked firing in pyramidal neurons, and $C_{a v}|.2-\alpha| c$ expression was increased in the mPFC, of Tg rats. (a) Blockade of the L-channels by $40 \mathrm{nM}$ diltiazem did not significantly reduce evoked firing of naive non- $\mathrm{Tg}$ neurons. Current-spike number relationships revealed that evoked firing of naive Tg neurons was significantly reduced following blockade of the L-channels by $40 \mathrm{nM}$ diltiazem (mixed model ANOVA with post-hoc NewmanKeuls, $* * p<0.01$ and ${ }^{*} * * p<0.00$ I). Insert: To illustrate that instrumentation of the transgenic animals did not influence baseline excitability, shown are current-evoked spike relationships for depolarizing current pulses (+25 to 200 pA) obtained from neurons of treatment-naive non-Tg rats and SAL-Yoked Tg rats (without blockade of L-channels). (b) Cavl.2- $\alpha \mid c$ immunoreactivity. Shown are representative photomicrographs of layers 5/6 within the prelimbic (PrL) mPFC taken from SAL-Yoked non- $\mathrm{Tg}$ (left) and $\mathrm{Tg}$ (middle) rats (scale bar $=25 \mu \mathrm{m}$ ). The stereotaxic map (right) is relative to Bregma (redrawn from (Paxinos and Watson, 1998)). (c) Tg rats had a significantly greater number of Ca l l.2-immunoreactive cells within PrL-mPFC layers 5/6. Student's t-test; ***** $<0.00$ I.

Several measures of excitability differed among test conditions. The RMP was depolarized in the Tg state and following cocaine, which enhances excitability to excitatory inputs (Dougherty et al, 2012). Acute perfusion with the HIV-1 protein Tat also depolarizes the RMP and this effect is exacerbated in rats with a history of COC-SA (Wayman et al, 2015a). These outcomes may reflect a downregulation of $K_{2 P}$ channels, as these channels are critical mediators of RMP (Braun, 2012). Unique to COC-SA Tg rats was a decrease in the AHP. As AHP participates in inter-spike interval timing, AHP reduction can increase spiking rates. Calcium-activated $\mathrm{K}^{+}$channels regulate the AHP (Sah and Faber, 2002); therefore, these channels may be downregulated by HIV-1 proteins and/or chronic cocaine. PN from $\mathrm{Tg}$ rats demonstrated several features of hyper-excitability, including increased spontaneous and evoked firing, and a greater tendency for depolarization-induced inactivation. These outcomes are consistent with increased $\mathrm{Ca}^{2+}$ influx, which, when excessive, can interfere with the capacity of neurons to repolarize and thus profoundly interfere with normal function. High voltage-activated L-channel $\mathrm{Ca}_{\mathrm{v}} 1.2-\alpha 1 \mathrm{c}$ protein was overexpressed in the $\mathrm{mPFC}$ of $\mathrm{Tg}$ rats, both in the number of $\mathrm{Ca}_{\mathrm{v}} 1.2$-ir PN and in the apparent level of expression within PN. Increased expression of $\mathrm{Ca}_{\mathrm{v}} 1.2-\alpha 1 \mathrm{c}$ also occurs in the mPFC of rats following intraventricular injections of HIV-1 Tat (Wayman et al, 2012). High voltageactivated L-channels are responsible for conducting large $\mathrm{Ca}^{2+}$ currents into the neuron, and increasing the number and/or activity of these channels profoundly influences neuronal excitability. PN from COC-SA Tg rats trended toward exhibiting the greatest spiking rate. We previously determined from rats which self-administered $20 \mathrm{mg} / \mathrm{kg} /$ day of cocaine, that PN exhibited an additive enhancement of spiking by cocaine and HIV-1 Tat (Wayman et al, 2015a). Rat in the present study self-administered $\sim 1 \mathrm{mg} / \mathrm{kg} / \mathrm{day}$ of cocaine, and we hypothesize that with higher doses of 
cocaine PN firing would also demonstrate additivity for cocaine and HIV-1 proteins. The electrophysiological profile of $\mathrm{PN}$ in $\mathrm{Tg}$ rats without cocaine was consistent with L-channel overactivation, and the effects were mitigated by blocking the L-channels with diltiazem only in Tg rats. We previously revealed in Sprague-Dawley rats that the same diltiazem concentration (ie, $40 \mathrm{nM}$ ) is sufficient to block Tatinduced enhancements in isolated $\mathrm{Ca}^{2+}$ potentials in mPFC PN without altering baseline firing (Napier et al, 2014). It is plausible that the ability of diltiazem to attenuate the baseline profiles of $\mathrm{mPFC} \mathrm{PN}$ in $\mathrm{Tg}$ rats reflects the consequences of chronically expressed HIV-1 proteins, including Tat. COCSA Tg rats also presented the largest percentage of neurons exhibiting posthyperpolarization rebound firing. Low voltage-activated T-Type and L-Type $\left(\mathrm{Ca}_{\mathrm{v}} 1.3\right) \mathrm{Ca}^{2+}$ channels are thought to mediate such rebound spiking (Markram et al, 1997; Markram and Sakmann, 1994; Lipscombe, 2002). It would be interesting to observe whether these channels are also upregulated in Tg rats and after cocaine.

HIV-1 proteins are critically involved in HIV-associated neuropathology (Nath, 2002) and the neurotoxicity of these proteins is amplified by drug abuse (Aksenov et al, 2006; Meyer et al, 2013; Wayman et al, 2015a, b). The present study revealed that overexcitation of $\mathrm{MPFC}$ PN was associated with the HIV-1 Tg brain state and COC-SA. The neuronal hyperresponsivity likely reflects alterations in mechanisms that mediate subthreshold and suprathreshold excitability, including L-type $\mathrm{Ca}^{2+}$ channels that are common to both chronic conditions. Changes in PN excitability did not influence cocaine intake, ie, Tg and non-Tg rats were similar, suggesting that drug reward-motivated behavior remained intact. The hyper-excitability may, however, underpin the enhanced psychological distress and neurological decline reported for the comorbid individual (Gibbie et al, 2007; Meade et al, 2011; Meyer et al, 2013). Outcomes from Tg rats also indicate that mPFC PN remain vulnerable to HIVassociated dysregulation even when viral replication is controlled, thus providing a pathophysiological explanation for the persistent behavioral deficits reported for these individuals (Meyer et al, 2013; Connolly et al, 2014).

\section{FUNDING AND DISCLOSURE}

The authors declare no conflict of interest.

\section{ACKNOWLEDGMENTS}

Studies overviewed in this manuscript were supported in part by USPHSG's DA033206 and DA033882, the Center for Compulsive Behavior and Addiction at Rush University Medical Center, and the Chicago Developmental Center for AIDS Research P30A1082151.

\section{REFERENCES}

Aksenov MY, Aksenova MV, Nath A, Ray PD, Mactutus CF, Booze RM (2006). Cocaine-mediated enhancement of Tat toxicity in rat hippocampal cell cultures: the role of oxidative stress and D1 dopamine receptor. Neurotoxicology 27: 217-228.

Brailoiu GC, Brailoiu E, Chang JK, Dun NJ (2008). Excitatory effects of human immunodeficiency virus 1 Tat on cultured rat cerebral cortical neurons. Neuroscience 151: 701-710.
Braun AP (2012). Two-pore domain potassium channels: variation on a structural theme. Channels (Austin) 6: 139-140.

Connolly CG, Bischoff-Grethe A, Jordan SJ, Woods SP, Ellis RJ, Paulus MP et al (2014). Altered functional response to risky choice in HIV infection. Plos One 9: e111583.

Dougherty KA, Islam T, Johnston D (2012). Intrinsic excitability of CA1 pyramidal neurones from the rat dorsal and ventral hippocampus. J Physiol 590: 5707-5722.

Ferris MJ, Mactutus CF, Booze RM (2008). Neurotoxic profiles of HIV, psychostimulant drugs of abuse, and their concerted effect on the brain: current status of dopamine system vulnerability in NeuroAIDS. Neurosci Biobehav Rev 32: 883-909.

Gibbie T, Hay M, Hutchison CW, Mijch A (2007). Depression, social support and adherence to highly active antiretroviral therapy in people living with HIV/AIDS. Sex Health 4: 227-232.

Glossmann H, Linn T, Rombusch M, Ferry DR (1983). Temperature-dependent regulation of d-cis- $[3 \mathrm{H}]$ diltiazem binding to $\mathrm{Ca} 2+$ channels by 1,4-dihydropyridine channel agonists and antagonists. FEBS Lett 160: 226-232.

Goldstein RZ, Volkow ND (2002). Drug addiction and its underlying neurobiological basis: neuroimaging evidence for the involvement of the frontal cortex. Am J Psychiatry 159: $1642-1652$.

Gonzalez-Scarano F, Martin-Garcia J (2005). The neuropathogenesis of AIDS. Nat Rev Immunol 5: 69-81.

Grace AA, Bunney BS (1986). Induction of depolarization block in midbrain dopamine neurons by repeated administration of haloperidol: analysis using in vivo intracellular recording. J Pharmacol Exp Ther 238: 1092-1100.

Haughey NJ, Mattson MP (2002). Calcium dysregulation and neuronal apoptosis by the HIV-1 proteins Tat and gp120. J Acquir Immune Defic Syndr 31(Suppl 2): S55-S61.

Hauser KF, El-Hage N, Stiene-Martin A, Maragos WF, Nath A, Persidsky Y et al (2007). HIV-1 neuropathogenesis: glial mechanisms revealed through substance abuse. J Neurochem 100: $567-586$.

Heaton RK, Clifford DB, Franklin DR Jr, Woods SP, Ake C, Vaida $\mathrm{F}$ et al (2010). HIV-associated neurocognitive disorders persist in the era of potent antiretroviral therapy: CHARTER Study. Neurology 75: 2087-2096.

Heaton RK, Franklin DR, Ellis RJ, McCutchan JA, Letendre SL, Leblanc $S$ et al (2011). HIV-associated neurocognitive disorders before and during the era of combination antiretroviral therapy: differences in rates, nature, and predictors. J Neurovirol 17: 3-16.

Jones M, Olafson K, Del Bigio MR, Peeling J, Nath A (1998). Intraventricular injection of human immunodeficiency virus type 1 (HIV-1) tat protein causes inflammation, gliosis, apoptosis, and ventricular enlargement. J Neuropathol Exp Neurol 57: 563-570.

Kolb B, Gibb R (2015). Plasticity in the prefrontal cortex of adult rats. Front Cell Neurosci 9: 15.

Lipscombe D (2002). L-type calcium channels - Highs and new lows. Circ Res 90: 933-935.

Markram H, Lubke J, Frotscher M, Sakmann B (1997). Regulation of synaptic efficacy by coincidence of postsynaptic APs and EPSPs. Science 275: 213-215.

Markram H, Sakmann B (1994). Calcium transients in dendrites of neocortical neurons evoked by single subthreshold excitatory postsynaptic potentials via low-voltage-activated calcium channels. Proc Natl Acad Sci USA 91: 5207-5211.

McBride JL, During MJ, Wuu J, Chen EY, Leurgans SE, Kordower JH. Structural and functional neuroprotection in a rat model of Huntington's disease by viral gene transfer of GDNF. Exp Neurol, (2003) 181: 213-223.

McIntosh S, Sexton T, Pattison LP, Childers SR, Hemby SE (2015). Increased sensitivity to cocaine self-administration in HIV-1 transgenic rats is associated with changes in striatal dopamine transporter binding. J Neuroimmune Pharmacol 10: 493-505. 
Meade CS, Conn NA, Skalski LM, Safren SA (2011). Neurocognitive impairment and medication adherence in HIV patients with and without cocaine dependence. J Behav Med 34: 128-138.

Meyer AC, Bardo MT (2015). Amphetamine self-administration and dopamine function: assessment of gene $\mathrm{x}$ environment interactions in Lewis and Fischer 344 rats. Psychopharmacology (Berl) 232: 2275-2285.

Meyer VJ, Rubin LH, Martin E, Weber KM, Cohen MH, Golub ET et al (2013). HIV and recent illicit drug use interact to affect verbal memory in women. J Acquir Immune Defic Syndr 63: 67-76.

Napier TC, Chen L, Kashanchi F, Hu XT (2014). Repeated cocaine treatment enhances HIV-1 Tat-induced cortical excitability via over-activation of L-type calcium channels. J Neuroimmune Pharmacol 9: 354-368.

Nath A (2002). Human immunodeficiency virus (HIV) proteins in neuropathogenesis of HIV dementia. J Infect Dis 186: S193-S198.

Nath A, Haughey NJ, Jones M, Anderson C, Bell JE, Geiger JD (2000). Synergistic neurotoxicity by human immunodeficiency virus proteins Tat and gp120: protection by memantine. Ann Neurol 47: 186-194.

Paxinos G, Watson C (1998). The Rat Brain in Stereotaxic Coordinates. Academic Press: New York, USA.

Picetti R, Caccavo JA, Ho A, Kreek MJ (2012). Dose escalation and dose preference in extended-access heroin self-administration in Lewis and Fischer rats. Psychopharmacology (Berl) 220: 163-172.

Rao JS, Kim HW, Kellom M, Greenstein D, Chen M, Kraft AD et al (2011). Increased neuroinflammatory and arachidonic acid cascade markers, and reduced synaptic proteins, in brain of HIV-1 transgenic rats. J Neuroinflammation 8: 101-114.
Reid W, Sadowska M, Denaro F, Rao S, Foulke J Jr, Hayes N et al (2001). An HIV-1 transgenic rat that develops HIV-related pathology and immunologic dysfunction. Proc Natl Acad Sci USA 98: 9271-9276.

Sah P, Faber ES (2002). Channels underlying neuronal calciumactivated potassium currents. Prog Neurobiol 66: 345-353.

Thompson PM, Dutton RA, Hayashi KM, Toga AW, Lopez OL, Aizenstein HJ et al (2005). Thinning of the cerebral cortex visualized in HIV/AIDS reflects CD4+ T lymphocyte decline. Proc Natl Acad Sci USA 102: 15647-15652.

Tozzi V, Balestra P, Bellagamba R, Corpolongo A, Salvatori MF, Visco-Comandini U et al (2007). Persistence of neuropsychologic deficits despite long-term highly active antiretroviral therapy in patients with HIV-related neurocognitive impairment: prevalence and risk factors. J Acquir Immune Defic Syndr 45: 174-182.

Wayman WN, Chen L, Napier TC, Hu X-T (2015a). Cocaine selfadministration enhances excitatory responses of pyramidal neurons in the rat medial prefrontal cortex to human immunodeficiency virus-1 Tat. Eur J Neurosci 41: 1195-1206.

Wayman WN, Chen L, Persons AL, Napier TC (2015b). Cortical consequences of HIV-1 Tat exposure in rats are enhanced by chronic cocaine. Curr HIV Res 13: 80-87.

Wayman WN, Dodiya HB, Persons AL, Kashanchi F, Kordower JH, $\mathrm{Hu} \mathrm{X}-\mathrm{T}$ et al (2012). Enduring cortical alterations after a single in vivo treatment of HIV-1 Tat. Neuroreport 23: 825-829.

Wen Y, Li B, Han F, Wang E, Shi Y (2012). Dysfunction of calcium/ calmodulin/CaM kinase II alpha cascades in the medial prefrontal cortex in post-traumatic stress disorder. Mol Med Rep 6: 1140-1144.

World Health Organization, Joint United Nations Programme on HIV/AIDS (2009). AIDS Epidemic Update 2009. 